Journal of Agricultural Sciences
(Tarim Bilimleri Dergisi)

\title{
Genetic Diversity and Phylogenetic Relationships of Turkish Local Popcorn (Zea mays everta) Populations analyzed by Simple Sequence Repeats (SSRs) Markers
}

\author{
Gulay ZULKADIR $^{\mathbf{a}^{*}} \mathbb{D}$, Leyla IDIKUT ${ }^{\mathrm{b}}$ (D) \\ ${ }^{a}$ Department of Organic Agriculture Management, Applied Technology and Management School of Silifke, Mersin University, Silifke, Mersin, TURKEY \\ ${ }^{b}$ Department of Crop Field, Faculty of Agriculture, Kahramanmaras Sutcu Imam University, Kahramanmaras, TURKEY \\ ARTICLE INFO \\ Research Article \\ Corresponding Author: Gulay ZULKADIR, E-mail: gulayzulkadir@gmail.com \\ Received: 30 September 2019 / Revised: 06 December 2019 / Accepted: 26 December 2019 / Online: 31 May 2021
}

\section{ABSTRACT}

Maize (Zea mays everta) is preferred as a good dietary in Turkey and it is important to know its genetic diversity to improve the yield. Genetic markers are very important in determining genetic diversity in popcorn populations. The aim of this study was to evaluate the genetic diversity of landraces popcorn populations by simple sequence repeats (SSR) markers. A hundred seventy five accessions of popcorn from thirty five populations grown in Turkey were analyzed using twenty SSR markers.

As a result of molecular analysis, 65 of 66 alleles obtained were showed polymorphisms and the polymorphism rate was $98.5 \%$. The average number of alleles for each SSR loci was 3.3, and this the number of alleles varied from 1 to 5 . The average the polymorphism information content (PIC) value was calculated to be 0.57 for SSR locus ranging from 0.00 to 0.89 . The number and percentage of polymorphic loci of the genotypes were determined to vary between $29 / 47 \%$ and $43.94 / 71.21 \%$ and the mean values were calculated as 39.114 and $59.265 \%$ respectively. The value of genetic change in the phylogenetic tree obtained from landraces popcorn populations was determined as 0.05 , and the genetic difference among genotypes varied from 14.7 to $97.1 \%$. Among the markers used in the study, it was observed that code 'phi064' was the most effective marker for determining genetic diversity in popcorn and the highest allele frequency also on this marker was obtained.

Keywords: Genetic diversity, Landrace, Molecular characterization, Popcorn, SSR

(C) Ankara University, Faculty of Agriculture

\section{Introduction}

Maize is the major cereal growing all over the world and it is a considerable cereal ranked as the third in world cereal production after wheat and rice (Adjanohoun et al. 2011). One of the commercially produced plants in the corn variety is popcorn. Popcorn (Zea mays everta) can be easily distinguished by plant and seed characteristics among other maize varieties.

Popcorn is preferred in terms of its rich nutritional content, vitamins and minerals. Also, corn is a good dietary product with stomach acidic absorption properties, low calorie and whole grain. The multi-purpose use of the corn has led to intensive research on the plant. Through the breeding studies in popcorn, different breeding methods are applied to ensure suitability for the purpose of efficiency such as adaptation, aquaculture and quality criteria. In order to obtain the necessary variation in breeding activities, registered varieties, local varieties and wild relatives should be screened and the appropriate genes should be transferred to the cultivars by improved techniques. The success in plant breeding is first and foremost provided by an efficient, accurate and rapid selection (Frankel 1972).

Maize spread from Central America to the other regions of the world. In course of time, it adapted to the extreme climatic conditions and thus, now it is characterized by a high degree of genetic variability. Morphological characterization is highly affected by the environment conditions therefore it indicates variability (Aci et al. 2013). On the other hand, molecular characterization is not affected by the environmental conditions and it provides valuable genetic information (Gauthier et al. 2002). In order to reveal the crucial genetic information, molecular markers such as microsatellites (SSR) are used (Yao et al. 2008; Liu et al. 2009; Eschholz et al. 2010) and have been very helpful for determining the diversity.

The aim of this study was to analyze the genetic diversity among different popcorn populations via SSRs markers on the samples collected from the different regions in Turkey. 


\section{Material and Methods}

\subsection{Plant material}

In this study, one hundred and seventy five accessions of popcorn were used from total 35 population that grown in various cities of Turkey including 34 landraces populations and one standard variety (Table 1). This collection was provided by Department of Plant Gene Resources in Ege Agricultural Research and Antalya West Mediterranean Agricultural Research Institute (Nermin Cin 98 as Standard variety).

Table 1- Genotype No, Registration No, Province, Region, Altitude and Material Color Information of the material used in the research

\begin{tabular}{|c|c|c|c|c|c|c|c|c|c|c|c|}
\hline 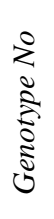 & 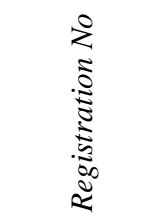 & $\begin{array}{l}\stackrel{\Xi}{\Xi} \\
0 \\
0 \\
0\end{array}$ & $\begin{array}{l}\tilde{\Xi} \\
\stackrel{\varpi}{\infty} \\
\approx \\
\approx\end{array}$ & $\underset{\mathbb{Z}}{\stackrel{\Xi}{\Xi}}$ & 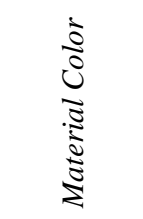 & 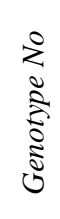 & 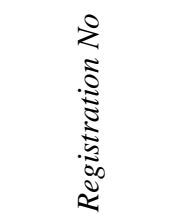 & 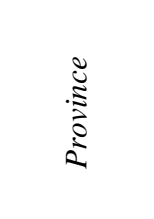 & $\begin{array}{l}\tilde{s} \\
\tilde{\infty} \\
\tilde{2}\end{array}$ & 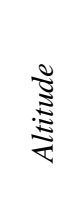 & 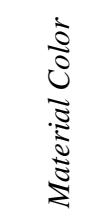 \\
\hline 1 & TR79913* & Canakkale & Biga & 40 & Yellow & 19 & TR78053* & Kutahya & Simav & 950 & Yellow \\
\hline 2 & TR79947* & Balikesir & Gonen & 120 & Red & 20 & TR78181* & Usak & Sivas & 970 & Yellow \\
\hline 3 & TR79947* & Balikesir & Gonen & 120 & Yellow & 21 & TR76375* & Diyarbakir & Cungus & 939 & Yellow \\
\hline 4 & TR79947* & Balikesir & Gonen & 120 & Pied & 22 & TR73761* & Eskisehir & Gunyuzu & 916 & Yellow \\
\hline 5 & TR79987* & Balikesir & Bigadic & 437 & Dark Red & 23 & TR73698* & Eskisehir & Beylikova & 789 & Yellow \\
\hline 6 & TR79987* & Balikesir & Bigadic & 437 & Orange & 24 & TR74311* & Kayseri & Hacilar & 1479 & Yellow \\
\hline 7 & TR73836* & Eskisehir & Gunyuzu & 991 & Yellow & 25 & TR85132* & Tokat & Erbaa & --- & Yellow \\
\hline 8 & TR73836* & Eskisehir & Gunyuzu & 991 & Orange & 26 & TR37977* & Tokat & Merkez & 560 & $\begin{array}{l}\text { Light } \\
\text { Yellow }\end{array}$ \\
\hline 9 & TR79988* & Balikesir & Bigadic & 437 & White & 27 & $\begin{array}{l}\text { Ordu - } \\
\text { Dogulu }\end{array}$ & Ordu & Dogulu & --- & Red \\
\hline 10 & TR79988* & Balikesir & Bigadic & 437 & Yellow & 28 & Konya Pop & Konya & --- & --- & Red \\
\hline 11 & TR73746* & Eskisehir & Gunyuzu & 916 & Orange & 29 & $\begin{array}{c}\text { Nermin } \\
\text { Cin** }\end{array}$ & --- & --- & --- & Yellow \\
\hline 12 & TR73746* & Eskisehir & Gunyuzu & 916 & $\begin{array}{l}\text { Light } \\
\text { Orange }\end{array}$ & 30 & Tokat Erbaa & Tokat & Erbaa & --- & Yellow \\
\hline 13 & TR39601* & Artvin & Ardanuc & 1300 & Red & 31 & $\begin{array}{c}\text { Samsun } \\
\text { Bafra }\end{array}$ & Samsun & Bafra & --- & Orange \\
\hline 14 & TR79932* & Canakkale & Can & 103 & White & 32 & $\begin{array}{c}\text { Ordu- } \\
\text { Akpinar }\end{array}$ & Ordu & Akpinar & --- & $\begin{array}{l}\text { Light } \\
\text { Yellow }\end{array}$ \\
\hline 15 & TR78115* & Afyon & İncehisar & 1140 & Yellow & 33 & $\begin{array}{c}\text { Ordu- } \\
\text { Kovanli }\end{array}$ & Ordu & Kovanli & --- & Yellow \\
\hline 16 & TR76741* & Tekirdag & Sarkoy & 120 & Dark Red & 34 & TR54215* & Mugla & Fethiye & 1130 & Yellow \\
\hline 17 & TR38027* & Amasya & Sukuova & 400 & $\begin{array}{l}\text { White/ } \\
\text { Yellow }\end{array}$ & 35 & TR54215* & Mugla & Fethiye & 1130 & White \\
\hline 18 & TR74236* & Kastamonu & Taskopru & 896 & Orange & & & & & & \\
\hline
\end{tabular}

*, Genotype codes obtained from the Plant Gene Resources Department of Ege Agricultural Research Institute; **, Standard variety. Others populations was collected from various parts of Turkey

\subsection{DNA extraction}

DNA isolation and SSR analysis were carried out in Kahramanmaras Sutcu Imam University, Faculty of Agriculture, Department of Field Crops Laboratory and University - Industry - Public Cooperation Development, Application and Research Center (USKIM). 
Maize seeds were grown in the greenhouse. Five plants (at 4-5 leave stage) were taken randomly from each population and stored at $-80^{\circ} \mathrm{C}$. Single-plant samples were ground to powder in liquid nitrogen using a mortar and pestle. A total genomic DNA was extracted following a modified procedure by Doyle \& Doyle (1987).

\subsection{SSR analysis}

Twenty SSR primer pairs of maize were selected from two of each chromosome, however it was used in previous studies and reported to be effective (Table 2).

Table 2- Name of the SSR markers, Chromosome locations, DNA Sequences and Base Numbers used in the study

\begin{tabular}{|c|c|c|c|c|c|c|c|}
\hline 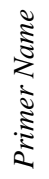 & $\begin{array}{l}\tilde{D} \\
\vdots \\
\vdots \\
\vdots \\
\vdots \\
\vdots \\
\vdots\end{array}$ & & DNA Sequence $\left(5^{\prime} \rightarrow 3^{\prime}\right)$ & 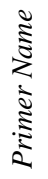 & $\begin{array}{l}\cong \\
\vdots \\
\vdots \\
\vdots \\
\vdots \\
\vdots \\
\vdots\end{array}$ & & DNA Sequence $\left(5^{\prime} \rightarrow 3^{\prime}\right)$ \\
\hline 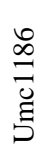 & 6.02 & $\mathrm{R}$ & $\begin{array}{l}\text { TCAAGAACATAATAGGAGGCCCAC } \\
\text { AGCCAGCTTGATCTTTAGCATTTG }\end{array}$ & $\frac{n}{a}$ & 8.00 & $\mathrm{R}$ & $\begin{array}{l}\text { GCAACGTACCGTACCTTTCCGA } \\
\text { ACGCTGCATTCAATTACCGGGAAG }\end{array}$ \\
\hline 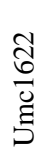 & 2.00 & $\mathrm{~F}$ & $\begin{array}{l}\text { CGCTACAAATCCTACTGGTGCTTT } \\
\text { CCTCGGATTTTCCAAAACATTTCT }\end{array}$ & 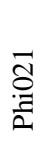 & 4.00 & $\mathrm{~F}$ & TTCCATTCTCGTGTTCTTGGAGTGGTCCA \\
\hline 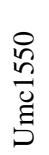 & 4.03 & $\mathrm{R}$ & $\begin{array}{l}\text { CGGGGTAATTGGGTACATAACCTC } \\
\text { GTGCCTCCAACGCCTAGTTTTT }\end{array}$ & సิ & 9.00 & $\mathrm{~F}$ & TGCGCACCAGCGACTGACC \\
\hline 角 & 1.03 & $\mathrm{~F}$ & $\begin{array}{c}\text { CCGATCGGCTTTATCACTGTTTAGC } \\
\text { ATGCACCATTCTAGCACTATAGCAACACT }\end{array}$ & ָิ & 9.03 & $\begin{array}{l}\mathrm{F} \\
\mathrm{R}\end{array}$ & $\begin{array}{l}\text { CACAGCACGTTGCGGATTTCTCT } \\
\text { GCGTACGTACGACGAAGACAC }\end{array}$ \\
\hline 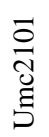 & 3.00 & $\mathrm{~F}$ & $\begin{array}{l}\text { CCCGGCTAGAGCTATAAAGCAAGT } \\
\text { CTAGCTAGTTTGGTGCGTGGTGAT }\end{array}$ & 突 & 7.00 & $\mathrm{R}$ & TAGCGACAGGATGGCCTCTTCT \\
\hline$\frac{n}{2}$ & 4.11 & $\mathrm{~F}$ & $\begin{array}{c}\text { GGACTACATCACGCCGGAGAT } \\
\text { TTTGGGAGAACAATCGGTTCTGTA }\end{array}$ & 龸 & 1.00 & $\mathrm{~F}$ & $\begin{array}{l}\text { CCGAATTGAAATAGCTGCGAGAACCT } \\
\text { ACAATGAACGGTGGTTATCAACACGC }\end{array}$ \\
\hline$\frac{1}{2}$ & 9.02 & $\mathrm{R}$ & $\begin{array}{l}\text { CGTTGGCGACCAGGGTGCGTTGGAT } \\
\text { TGCAACAGCCATTCGATCATCAAAC }\end{array}$ & $\underset{\substack{1 \\
\infty}}{\stackrel{0}{2}}$ & 10.0 & $\mathrm{~F}$ & $\begin{array}{l}\text { AGAAGGAATCCGATCCATCCAAGC } \\
\text { CACCCGTACTTGAGGAAAACCC }\end{array}$ \\
\hline 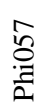 & 7.01 & $\begin{array}{l}\mathrm{F} \\
\mathrm{R}\end{array}$ & $\begin{array}{l}\text { CTCATCAGTGCCGTCGTCCAT } \\
\text { CAGTCGCAAGAAACCGTTGCC }\end{array}$ & 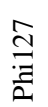 & 2.00 & $\mathrm{~F}$ & $\begin{array}{l}\text { ATATGCATTGCCTGGAACTGGAAGGA } \\
\text { AАTTCAAACACGCCTCCCGAGTGT }\end{array}$ \\
\hline 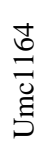 & 4.01 & $\mathrm{R}$ & $\begin{array}{l}\text { AAATAAACGCTCCAAAGAAAGCAA } \\
\text { GCACGTGTGTGTGTGTTGTTTTTA }\end{array}$ & 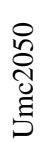 & 3.00 & $\mathrm{~F}$ & CTGGATCTCGGCATGGTCTT \\
\hline 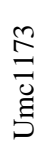 & 4.09 & $\mathrm{R}$ & $\begin{array}{l}\text { ATCCGCCAAAAAGGGGAAAA } \\
\text { TAGAAGTAGCACACGCGCCG }\end{array}$ & 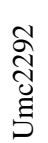 & 5.00 & $\mathrm{~F}$ & AGCAGAAGAGGACAAACCAGATTC \\
\hline
\end{tabular}

The total volume of PCR mixture was $20 \mu \mathrm{L}$ containing $2 \mu \mathrm{L}$ ddH ${ }_{2} \mathrm{O}, 3.5 \mu \mathrm{L}$ 10X PCR buffer $\left(\mathrm{Mg}^{+2}\right.$ added $), 1.2 \mu \mathrm{L}$ dNTP $(5 \mathrm{mM}), 4 \mu \mathrm{L}$ F primer $(20 \mu \mathrm{M}), 4 \mu \mathrm{L}$ R primer $(20 \mu \mathrm{M}), 5 \mu \mathrm{L}$ Genomic DNA (100 ng $), 0.3 \mu \mathrm{L}^{\mathrm{DNA}}$ Taq polymerase $\left(5 \mathrm{U}^{-1}\right.$, Fermantes). 
The PCR reaction was performed in a thermal cycler (Eppendorf Mastercycler Gradient) using an initial $94{ }^{\circ} \mathrm{C}$ denaturing step for $5 \mathrm{~min}$ followed by 34 cycles of [denaturation at $94{ }^{\circ} \mathrm{C}$ for $1 \mathrm{~min}$, annealing for $1 \mathrm{~min}$ at the primer's annealing temperature, extension at $72{ }^{\circ} \mathrm{C}$ for $\left.1 \mathrm{~min}\right]$ and a final extension at $72{ }^{\circ} \mathrm{C}$ for $5 \mathrm{~min}$.

\subsection{Data analysis}

The presence (1) or absence (0) of PCR amplicons were coded. Then the data base was registered in an MS Excel spreadsheet in order to generate the analysis matrix. Genetic diversity parameters such as Polymorphism Information Content (PIC) as previously described by Laborda et al. (2005); polymorphism rate (P), number of alleles (Na), expected heterozygosity (He) and Shannon's phenetic index $(\mathrm{H})$ were estimated according to the method used by Nei 1972. Cluster analysis by Un-weighted Pair Group Method using Arithmetic Averages (UPGMA) were estimated according to the method used by Rohlf (1992) and genetic variation patterns among the maize genotypes were identified using PopGen32 (Population Genetic Analysis System, Version 32V) and MEGA (Molecular Evolutionary Genetics Analysis) 6 databases software, respectively.

\section{Results and Discussion}

\subsection{SSR polymorphism}

The SSR markers selected to analyze the genetic diversity of the maize accessions displayed different characteristic profiles. Thus, different numbers of polymorphic bands, percentage of polymorphism, Polymorphism Information Content (PIC), and expected heterozygosis have been generated using the SSR markers.

SSR markers used to molecular characterization, allele size, allele number and PIC values included 175 popcorn accessions occurring from 35 landraces popcorn population are reported in Table 3.

Table 3- Allele size, number and PIC value information of SSR markers used in molecular characterization of landraces popcorn populations

\begin{tabular}{cccccccc}
\hline Primer & Allel Size & $\begin{array}{c}\text { Number of } \\
\text { Alleles }\end{array}$ & PIC & Primer & Allel Size & $\begin{array}{c}\text { Number of } \\
\text { Alleles }\end{array}$ & PIC \\
\hline phi015 & $80-120$ & 4 & 0.88 & phi127 & $110-129$ & 3 & 0.40 \\
phi017 & $100-110$ & 4 & 0.76 & phi064 & $73-110$ & 5 & 0.89 \\
phi021 & $90-120$ & 4 & 0.87 & phi057 & $160-170$ & 3 & 0.57 \\
phi034 & $110-150$ & 4 & 0.70 & umc1550 & $140-280$ & 3 & 0.43 \\
umc2292 & $130-170$ & 5 & 0.89 & phi095 & $140-180$ & 3 & 0.56 \\
umc2101 & $150-180$ & 3 & 0.50 & phi022 & $150-180$ & 4 & 0.62 \\
umc2050 & $120-160$ & 4 & 0.82 & phi027 & $150-180$ & 4 & 0.87 \\
umc1622 & $40-90$ & 2 & 0.10 & umc1164 & $140-160$ & 2 & 0.09 \\
umc1186 & $220-240$ & 2 & 0.12 & umc1173 & $150-170$ & 3 & 0.64 \\
\hline
\end{tabular}

Nineteen of 20 SSR markers were noted to be polymorphic while one marker (phi084) was found to be monomorphic. Total numbers of alleles were detected as 66 and 65 of them were polymorphic. The polymorphism rate was calculated to be as high as $98.5 \%$.

Vivodík et al. (2017) characterizing 40 maize populations with 10 SSR markers determined that they had 65 alleles in total, and that the number of these alleles changed between 4-8 (mean 6.5 alleles) and the PIC value varied from 0.734 to 0.848 (mean PIC 0.810). Riberio et al. (2017) analyzed 48 single hybrid maize varieties commercially used in Brazil with 20 SSR markers and determined the average allele number as 9.8 and the average PIC value as 0.84 . Atanda \& Olaove (2017) analyzed 24 inbred maize lines with 20 SSR markers and identified total of 101 alleles and found that the average allele number was 5.5 and the average PIC was 0.46 . 
Allele numbers observed for each locus ranged from 1 (phi084) to 5 (phi064 and umc2292) with an average of 3.3 alleles per locus. The PIC value ranged from 0.00 (phi084) to 0.89 (umc2292 and phi064) with average value of 0.57 . Number and percentage of polymorphic loci of populations were observed to range from 29 and $43.94 \%$ (population 21 ) to 47 and $71.21 \%$ (population 13) with an average of 39.11 and 59.27\%, respectively (Table 4).

Table 4 - Number and percentage of polymorphic loci of maize populations by Nei 1973 method

\begin{tabular}{|c|c|c|c|c|c|c|c|c|}
\hline $\begin{array}{l}\sum_{2} \\
\vdots \\
\vdots \\
\vdots \\
\vdots \\
\vdots \\
0 \\
2\end{array}$ & 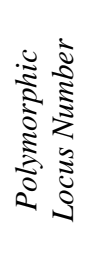 & 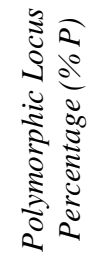 & $\begin{array}{l}\sum_{2}^{0} \\
\vdots \\
\vdots \\
\vdots \\
\vdots \\
0 \\
0 \\
2\end{array}$ & 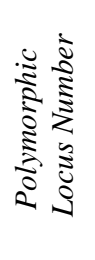 & 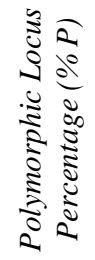 & 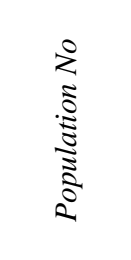 & 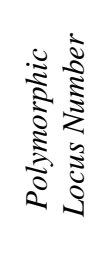 & 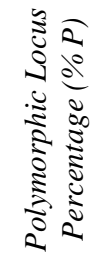 \\
\hline 1 & 40 & 60.61 & 13 & 47 & 71.21 & 25 & 39 & 59.09 \\
\hline 2 & 39 & 59.09 & 14 & 41 & 62.12 & 26 & 35 & 53.03 \\
\hline 3 & 38 & 57.58 & 15 & 38 & 57.58 & 27 & 41 & 62.12 \\
\hline 4 & 38 & 57.58 & 16 & 43 & 65.15 & 28 & 41 & 62.12 \\
\hline 5 & 39 & 59.09 & 17 & 38 & 57.58 & 29 & 41 & 62.12 \\
\hline 6 & 39 & 59.09 & 18 & 42 & 63.64 & 30 & 40 & 60.61 \\
\hline 7 & 38 & 57.58 & 19 & 44 & 66.67 & 31 & 36 & 54.55 \\
\hline 8 & 41 & 62.12 & 20 & 33 & 50.00 & 32 & 39 & 59.09 \\
\hline 9 & 37 & 56.06 & 21 & 29 & 43.94 & 33 & 39 & 59.09 \\
\hline 10 & 41 & 62.12 & 22 & 40 & 60.61 & 34 & 43 & 65.15 \\
\hline 11 & 37 & 56.06 & 23 & 36 & 54.55 & 35 & 44 & 66.67 \\
\hline 12 & 40 & 60.61 & 24 & 33 & 50.00 & Average & 39.114 & 59.265 \\
\hline
\end{tabular}

Molin et al. (2013) analyzed 48 local popcorn populations in Rio Grande do Sul and Parana in Brazil with 47 SSR markers and identified the polymorphic index as 78.3\%. Sharma et al. (2010) analyzed 48 local maize varieties in India with 42 SSR primers and as a result recorded $60 \%$ polymorphism rate.

\subsection{Genetic Differentiation}

The parameters $n_{a}, n_{e}, h, I$ and gen frequency revealed the genetic structure of the accessions (Table 5). Among polymorphic loci, the mean observed number of alleles $\left(\mathrm{n}_{\mathrm{a}}\right.$ ) varied from 1 (phi084) to 2 (all other alleles) and mean $\mathrm{n}_{\mathrm{a}}$ was 1.99 ; the mean effective number of alleles $\left(\mathrm{n}_{\mathrm{e}}\right.$ ) varied between 1.00 (phi084) and 1.99 (phi015-3) with an average of 1.65 for all accessions.

The total gen diversity (h) made according to Nei (1973) method was ranging from 0.0 to 0.5 with the average value of 0.36 . The Shannon's information index (I) varied between 0.00 and 0.69 with an average of 0.53 for all accessions. Finally, the gene frequency $(f)$ values; $f_{0}$ value ranged 0.00 to 0.98 with an average of 0.58 and the $f_{1}$ value ranged 0.0171 to 1.0000 with an average of 0.42 .

Polymorphism limit of alleles is accepted as $95 \%$, thus alleles are considered to be monomorphic if the frequency is $95 \%$ or more, however it is polymorphic when the allele frequency is below $95 \%$.

Accordingly, the allele frequencies in phi017-4 $\left(f_{0}=0.9714\right)$, phi034-1 $\left(f_{0}=0.9829\right)$, umc $2292-1\left(f_{0}=0.9714\right)$, umc2292-2 $\left(f_{0}=0.9714\right)$ and umc2050-4 $\left(f_{0}=0.9829\right)$ were $95 \%$, therefore, they were regarded as monomorphic. Polymorphic alleles are evaluated in their own, the allele frequency for 0 allele ranged from $f_{0}=0.0000$ (phi084) to $f_{0}=0.9314$ (phi064-5) and the allele frequency for 1 allele ranged from $f_{l}=0.0686$ (phi064-5) to $f_{l}=1.0000$ (phi084).

The investigated parameters, total genetic diversity $\left(\mathrm{H}_{t}\right)$, genetic diversity within the population $\left(\mathrm{H}_{s}\right)$, inter-population genetic differentiation $\left(\mathrm{G}_{s t}\right)$ and gene flow $\left(\mathrm{N}_{m}\right)$ revealed the genetic structure of the accessions (Table 6). 
Table 5- The mean observed number of alleles, mean effective number of alleles, genetic diversity according to Nei (1973) and Shannon's information index values of genetic variation of all loci

\begin{tabular}{|c|c|c|c|c|c|c|c|c|c|c|c|c|c|c|c|}
\hline \multirow{2}{*}{ Loci } & \multirow{2}{*}{ 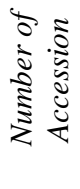 } & \multirow{2}{*}{$n_{a} *$} & \multirow{2}{*}{$n_{e}^{*}$} & \multirow{2}{*}{$h^{*}$} & \multirow{2}{*}{$I^{*}$} & \multicolumn{2}{|c|}{$\frac{\underline{\text { Gene }}}{\underline{\text { Frequency }}}$} & \multirow{2}{*}{ Loci } & \multirow{2}{*}{ 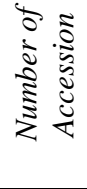 } & \multirow{2}{*}{$n_{a} *$} & \multirow{2}{*}{$n_{e}^{*}$} & \multirow{2}{*}{$h^{*}$} & \multirow{2}{*}{$I^{*}$} & \multicolumn{2}{|c|}{$\frac{\underline{\text { Gene }}}{\text { Frequency }}$} \\
\hline & & & & & & $\begin{array}{c}\text { Allel } \\
0\end{array}$ & $\begin{array}{c}\text { Allel } \\
1\end{array}$ & & & & & & & $\begin{array}{c}\text { Allel } \\
0\end{array}$ & $\begin{array}{c}\text { Allel } \\
1\end{array}$ \\
\hline phi015-1 & 175 & 20.00 & 12.127 & 0.175 & 0.319 & 0.903 & 0.097 & phi127-3 & 175 & 20.00 & 19.144 & 0.478 & 0.671 & 0.394 & 0.606 \\
\hline phi015-2 & 175 & 20.00 & 19.994 & 0.499 & 0.693 & 0.509 & 0.491 & phi064-1 & 175 & 20.00 & 13.243 & 0.245 & 0.410 & 0.857 & 0.143 \\
\hline phi015-3 & 175 & 20.00 & 19.999 & 0.500 & 0.693 & 0.497 & 0.503 & phi064-2 & 175 & 20.00 & 19.767 & 0.494 & 0.687 & 0.446 & 0.554 \\
\hline phi015-4 & 175 & 20.00 & 13.968 & 0.284 & 0.458 & 0.829 & 0.171 & phi064-3 & 175 & 20.00 & 18.760 & 0.467 & 0.660 & 0.629 & 0.371 \\
\hline phi017-1 & 175 & 20.00 & 14.115 & 0.291 & 0.467 & .823 & 0.177 & & 175 & 20.00 & 19.716 & 0.493 & 0.686 & 0.440 & 0.560 \\
\hline phi017-2 & 175 & 20.00 & 19.660 & 0.491 & 0.685 & .434 & 0.566 & phi064-5 & 175 & 20.00 & 11.464 & & & 0.931 & 0.069 \\
\hline phi017-3 & 175 & 20.00 & 19.054 & 0.475 & 0.668 & 0.389 & 0.611 & phi057-1 & 175 & 20.00 & 18.654 & 0.464 & 0.657 & 0.366 & 0.634 \\
\hline phi017-4 & 175 & 20.00 & 10.588 & 0.055 & 0.130 & 0.971 & 0.029 & phi057-2 & 175 & 20.00 & 19.890 & 0.497 & 0.690 & 0.463 & 0.537 \\
\hline phi021-1 & 175 & 20.00 & 19.600 & 0.489 & 0.683 & 0.571 & 0.429 & phi057-3 & 175 & 20.00 & 16.897 & 0.408 & 0.598 & 0.714 & 0.286 \\
\hline phi021-2 & 175 & 20.00 & 19.890 & 0.497 & 0.690 & .463 & 0.537 & umc1550-1 & 175 & 20.00 & 18.861 & 0.470 & 0.663 & 0.623 & 0.377 \\
\hline phi021-3 & 175 & 20.00 & 18.434 & 0.457 & 0.650 & .646 & 0.354 & umc1550-2 & 175 & & & & & 0.217 & 0.783 \\
\hline phi021-4 & 175 & 20.00 & 14.410 & 0.306 & 0.484 & .811 & 0.189 & & 175 & 20. & 17.959 & 0.443 & 0.635 & 0.669 & 0.331 \\
\hline phi034-1 & 175 & 20.00 & 10.349 & 0.033 & 0.087 & .983 & 0.017 & phi0 & 175 & 20.00 & 15.299 & 0.346 & & 0.777 & 0.223 \\
\hline phi034-2 & 175 & 20.00 & 19.054 & 0.475 & 0.668 & 0.611 & 0.389 & phi0s & 175 & 20.00 & 16.037 & 0.376 & 0.564 & 0.251 & 0.749 \\
\hline phi034-3 & 175 & 20.00 & 16.614 & 0.398 & 0.588 & 0.274 & 0.726 & phi0 & 175 & 20.00 & 15.743 & 0.365 & 0.551 & 0.760 & 0.240 \\
\hline phi034-4 & 175 & 20.00 & 11.595 & 0.137 & 0.265 & 0.926 & 0.074 & phi02 & 175 & 20.00 & 15.743 & 0.365 & 0.551 & 0.240 & 0.760 \\
\hline umc2292-1 & 175 & 20.00 & 10.588 & 0.055 & 0.130 & 0.971 & 0.029 & phi02 & 175 & 20.00 & 12.818 & 0.220 & 0.378 & 0.874 & 0.126 \\
\hline umc2292-2 & 175 & 20.00 & 10.588 & 0.055 & 0.130 & 0.971 & 0.029 & phi02 & 175 & 20.00 & 13.100 & 0.237 & 0.400 & 0.863 & 0.137 \\
\hline umc 2292-3 & 175 & 20.00 & 19.890 & 0.497 & 0.690 & 0.463 & 0.537 & phi022-4 & 175 & 20.00 & 19.854 & 0.496 & 0.690 & 0.543 & 0.457 \\
\hline umc2292-4 & 175 & 20.00 & 13.100 & 0.236 & 0.400 & 0.863 & 0.137 & phi027-1 & 175 & 20.00 & 18.202 & 0.451 & 0.643 & 0.657 & 0.343 \\
\hline umc2292-5 & 175 & 20.00 & 19.465 & 0.486 & 0.679 & 0.417 & 0.583 & phi027-2 & 175 & 20.00 & 19.854 & 0.496 & 0.690 & 0.457 & 0.543 \\
\hline umc2101-1 & 175 & 20.00 & 19.231 & 0.480 & 0.673 & 0.400 & 0.600 & phi027-3 & 175 & 20.00 & 19.231 & 0.480 & 0.673 & 0.600 & 0.400 \\
\hline umc2101-2 & 175 & 20.00 & 18.202 & 0.450 & 0.643 & 0.343 & 0.657 & phi027-4 & 175 & 20.00 & 15.596 & 0.359 & 0.544 & 0.766 & 0.234 \\
\hline umc 2101-3 & 175 & 20.00 & 11.595 & 0.137 & 0.265 & 0.926 & 0.074 & umc1164-1 & 175 & 20.00 & 13.100 & 0.237 & 0.400 & 0.137 & 0.863 \\
\hline umc2050-1 & 175 & 20.00 & 19.968 & 0.499 & 0.692 & 0.520 & 0.480 & umc1164-2 & 175 & 20.00 & 19.392 & 0.484 & 0.677 & 0.589 & 0.411 \\
\hline umc $2050-2$ & 175 & 20.00 & 19.716 & 0.492 & 0.686 & 0.440 & 0.560 & umc1173-1 & 175 & 20.00 & 19.767 & 0.494 & 0.687 & 0.446 & 0.554 \\
\hline umc 2050-3 & 175 & 20.00 & & & 0.677 & 0.589 & 0.411 & & 175 & & & & 0.681 & 0.577 & 0.423 \\
\hline umc 2050-4 & 175 & 20.00 & 10.349 & 0.033 & 0.087 & 0.983 & 0.017 & umc1173-3 & 175 & 20.00 & 19.968 & 0.499 & 0.692 & 0.520 & 0.480 \\
\hline umc1622-1 & 175 & 20.00 & 13.968 & 0.284 & 0.458 & 0.829 & 0.171 & umc1255-1 & 175 & 20.00 & 18.202 & 0.451 & 0.643 & 0.657 & 0.343 \\
\hline umc1622-2 & 175 & 20.00 & 11.464 & 0.127 & 0.250 & 0.069 & 0.931 & umc1255-2 & 175 & 20.00 & 19.231 & 0.480 & 0.673 & 0.400 & 0.600 \\
\hline umc1186-1 & 175 & 20.00 & 11.595 & 0.137 & 0.265 & 0.074 & 0.926 & umc1255-3 & 175 & 20.00 & 19.767 & 0.494 & 0.687 & 0.554 & 0.446 \\
\hline umc1186-2 & 175 & 20.00 & 13.676 & 0.268 & 0.440 & 0.840 & 0.160 & Average & 175 & 19.848 & 16.435 & 0.361 & 0.530 & 0.589 & 0.420 \\
\hline phi127-1 & 175 & 20.00 & 17.833 & 0.439 & 0.631 & 0.326 & 0.674 & $\begin{array}{l}\text { Standard } \\
\text { Error }\end{array}$ & 175 & 0.340 & 0.1543 & 0.193 & & & \\
\hline phi127-2 & 175 & 20.00 & 14.706 & 0.320 & 0.500 & 0.200 & 0.800 & & & & & & & & \\
\hline
\end{tabular}

$*$ na $=$ The mean observed number of alleles; $*$ ne $=$ The mean effective number of alleles (Kimura \& Crow 1964); $\mathrm{h}=\mathrm{Genetic}$ diversity according to Nei (1973); * I = Shannon's information index (Lewontin 1972).

While $\mathrm{H}_{t}$ varied between 0.0000 (phi084) and 0.5000 (phi015-3) with an average of $0.3606 ; \mathrm{H}_{s}$ ranged from 0.0000 (phi084) to 0.4206 (umc1255) and the average was $0.2391 . \mathrm{G}_{s t}$ values were identified to vary between 0.1290 (phi095-1) and 0.6706 (phi017-4) with an average of $0.3369 . \mathrm{N}_{m}$ values of genotypes ranged from 0.2456 to 3.3772 with a whole average value of 0.9840; the highest values on phi095-1 allele and the lowest on phi017-4 allele were observed. In general, it was observed that the phi095 coded marker was the most polymorphic marker in determining the diversity of genotypes for gene flow than other markers. On the other hand, phi084 coded marker revealed that it was not an effective marker for the determination of gene flow under this study.

In our study the genetic variation determined was lower than other that Vivodik et al. (2017) found for the 40 maize genotypes. Similarly, Zhang et al. (2016) examined 290 inbred maize lines by 201 SSR markers, and the diversity they determined was 0.70. The study accomplished by Tahir \& Maeruf (2016) on 9 corn genotypes with 18 SSR markers were reported that genetic diversity was between 0.20 and 0.82 . 
Table 6- Total genetic diversity, intra-population genetic diversity, inter-population genetic differentiation and gene flow data in determined SSR loci by Nei 1978 method

\begin{tabular}{|c|c|c|c|c|c|c|c|c|c|c|c|c|c|c|}
\hline Loci & $H_{t}$ & $H_{s}$ & $G_{s t}$ & $N_{m} *$ & Loci & $H_{t}$ & $H_{s}$ & $G_{s t}$ & $N_{m} *$ & Loci & $H_{t}$ & $H_{s}$ & $G_{s t}$ & $N_{m} *$ \\
\hline phi015-1 & 0.175 & 0.109 & 0.374 & 0.835 & umc2101-2 & 0.450 & 0.338 & 0.249 & 1.505 & umc1550-2 & 0.3400 & 0.2057 & 0.3949 & 0.7661 \\
\hline phi015-2 & 0.499 & 0.260 & 0.478 & 0.544 & umc2101-3 & 0.137 & 0.077 & 0.434 & 0.649 & umc $1550-3$ & 0.4432 & 0.2971 & 0.3295 & 10.174 \\
\hline phi015-3 & 0.500 & 0.306 & 0.387 & 0.790 & umc2050-1 & 0.499 & 0.306 & 0.386 & 0.793 & phi095-1 & 0.3464 & 0.3017 & 0.1290 & 33.772 \\
\hline phi015-4 & 0.284 & 0.128 & 0.549 & 0.410 & umc $2050-2$ & 0.492 & 0.315 & 0.359 & 0.889 & phi095-2 & 0.3764 & 0.3246 & 0.1378 & 31.297 \\
\hline phi017-1 & 0.291 & 0.187 & 0.357 & 0.900 & umc 2050-3 & 0.484 & 0.347 & 0.282 & 1.269 & phi095-3 & 0.3648 & 0.3154 & 0.1353 & 31.944 \\
\hline phi017-2 & 0.491 & 0.324 & 0.339 & 0.973 & umc 2050-4 & 0.033 & 0.022 & 0.321 & 1.054 & phi022-1 & 0.3648 & 0.1463 & 0.5990 & 0.3347 \\
\hline phi017-3 & 0.475 & 0.224 & 0.528 & 0.445 & umc1622-1 & 0.284 & 0.096 & 0.662 & 0.255 & phi022-2 & 0.2198 & 0.1143 & 0.4801 & 0.5415 \\
\hline phi017-4 & 0.055 & 0.018 & 0.670 & 0.245 & umc $1622-2$ & 0.127 & 0.054 & 0.570 & 0.376 & phi022-3 & 0.2367 & 0.1646 & 0.3046 & 11.413 \\
\hline phi021-1 & 0.489 & 0.352 & 0.281 & 1.277 & umc1186-1 & 0.137 & 0.054 & 0.601 & 0.331 & phi022-4 & 0.4963 & 0.3337 & 0.3276 & 10.261 \\
\hline phi021-2 & 0.497 & 0.342 & 0.310 & 1.110 & umc1186-2 & 0.268 & 0.123 & 0.540 & 0.424 & phi027-1 & 0.4506 & 0.2331 & 0.4826 & 0.5360 \\
\hline phi021-3 & 0.457 & 0.374 & 0.180 & 2.267 & phi127-1 & 0.439 & 0.274 & 0.375 & 0.831 & phi027-2 & 0.4963 & 0.2834 & 0.4289 & 0.6656 \\
\hline phi021-4 & 0.306 & 0.246 & 0.193 & 2.086 & phi127-2 & 0.320 & 0.214 & 0.328 & 1.021 & phi027-3 & 0.4800 & 0.3474 & 0.2762 & 13.103 \\
\hline phi034-1 & 0.033 & 0.013 & 0.593 & 0.343 & phi127-3 & 0.477 & 0.269 & 0.435 & 0.648 & phi027-4 & 0.3588 & 0.2469 & 0.3120 & 11.027 \\
\hline phi034-2 & 0.475 & 0.306 & 0.355 & 0.906 & phi064-1 & 0.244 & 0.137 & 0.440 & 0.636 & umc1164-1 & 0.2367 & 0.1829 & 0.2274 & 16.990 \\
\hline phi034-3 & 0.398 & 0.242 & 0.391 & 0.777 & phi064-2 & 0.494 & 0.310 & 0.370 & 0.848 & umc1164-2 & 0.4843 & 0.3474 & 0.2826 & 12.691 \\
\hline phi034-4 & 0.137 & 0.096 & 0.302 & 1.155 & phi064-3 & 0.466 & 0.297 & 0.363 & 0.875 & umc1173-1 & 0.4941 & 0.3977 & 0.1951 & 20.630 \\
\hline umc2292-1 & 0.055 & 0.036 & 0.341 & 0.965 & phi064-4 & 0.492 & 0.283 & 0.424 & 0.676 & umc1173-2 & 0.4881 & 0.3200 & 0.3444 & 0.9518 \\
\hline umc2292-2 & 0.055 & 0.027 & 0.505 & 0.488 & phi064-5 & 0.127 & 0.086 & 0.320 & 1.062 & umc1173-3 & 0.4992 & 0.3200 & 0.3590 & 0.8929 \\
\hline umc2292-3 & 0.497 & 0.306 & 0.384 & 0.802 & phi057-1 & 0.463 & 0.352 & 0.241 & 1.572 & umc1255-1 & 0.4506 & 0.3520 & 0.2188 & 17.848 \\
\hline umc 2292-4 & 0.236 & 0.146 & 0.381 & 0.809 & phi057-2 & 0.497 & 0.402 & 0.191 & 2.118 & umc1255-2 & 0.4800 & 0.3886 & 0.1905 & 21.250 \\
\hline umc 2292-5 & 0.486 & 0.292 & 0.398 & 0.755 & phi057-3 & 0.408 & 0.338 & 0.171 & 2.420 & umc1255-3 & 0.4941 & 0.4206 & 0.1488 & 28.597 \\
\hline \multirow[t]{2}{*}{ umc2101-1 } & 0.480 & 0.338 & 0.295 & 1.193 & umc1550-1 & 0.469 & 0.352 & 0.250 & 1.493 & Average & 0.3606 & 0.2391 & 0.3369 & 0.9840 \\
\hline & & & & & & & & & & Std. Error & 0.0238 & 0.0137 & & \\
\hline
\end{tabular}

$\mathrm{H}_{t}$, Total genetic diversity; $\mathrm{H}_{s}$, Genetic diversity within the population; $\mathrm{G}_{s t}$, Inter-population genetic differentiation; $* \mathrm{~N}_{\mathrm{m}}=\mathrm{Gene}_{\text {flow. }}$.g.; $\mathrm{N}_{m}=$ $0.5\left(1-\mathrm{G}_{s t}\right) / \mathrm{G}_{s t}$; (McDermott \& McDonald 1993)

\subsection{Genetic relationship and cluster analyses}

The amount of genetic change was determined as 0.05 and genetic differences among genotypes ranged between 14.7 and $97.1 \%$. According to our findings, while the lowest genetic distance was observed between 8.3 and 9.3 (Eskisehir-Ballkesir); 18.5 and 22.5 (Kastamonu-Eskisehir); 33.3 and 34.4 (Kovanli/Ordu-Mugla) coded genotypes with the average of 14.7\%, the highest genetic distance was determined between 15.3 and 26.4 (Afyon-Tokat) genotypes with $97.1 \%$.

Generally, when the genetic distance values are examined, variations among genotypes were observed to be very high and even among individuals of the same population, genetic differences were large.

A hundred seventy five genotypes were classified in two large clusters (Figure 1). While the first group had genotypes of population 15 (Incehisar/Afyon), the second group had all other genotypes. Then, second group divided into two sub-groups. The first branch of the second group had genotypes 35.2 and 1.2; the second branch of the second group had other genotypes with two sub-groups and then each group was divided into other sub-groups.

When the local populations were compared with the standard variety, it was relationship with all genotypes except for 15 number genotype. Because parental of standard varieties were obtained from populations collected from Turkey. This state is evidence that the local variety is selected from the country's populations and also the accuracy of the research conducted by us. It can be explained by cause moving to different region of the country with open pollination of popcorn genotypes as reason for being sub-groups under 2 groups and interconnected groups. Generally with telling, the distribution of local populations into groups was predominantly by provinces close to each other. In also, some populations obtained from different regions of Turkey were seen to be within the same group. Comertpay (2008), in analysis of the local corn according to the UPGMA method determined that the distribution of genotypes in groups does not show specific distribution; Warburton et al. (2005) reported that the elite corn lines were not divided into groups according to environmental factors and morphological characteristics. Our study 
was supported by these two studies, while populations except for 15 number population were being in the groups and subgroup, population of 15 number placed in a separate group.

The reasons of the high genetic diversity of genotypes are; the genotypes used are open fertilizer material, be grown in different places, be high adaptability and it can be concluded that hundreds of seeds can be taken from one plant.

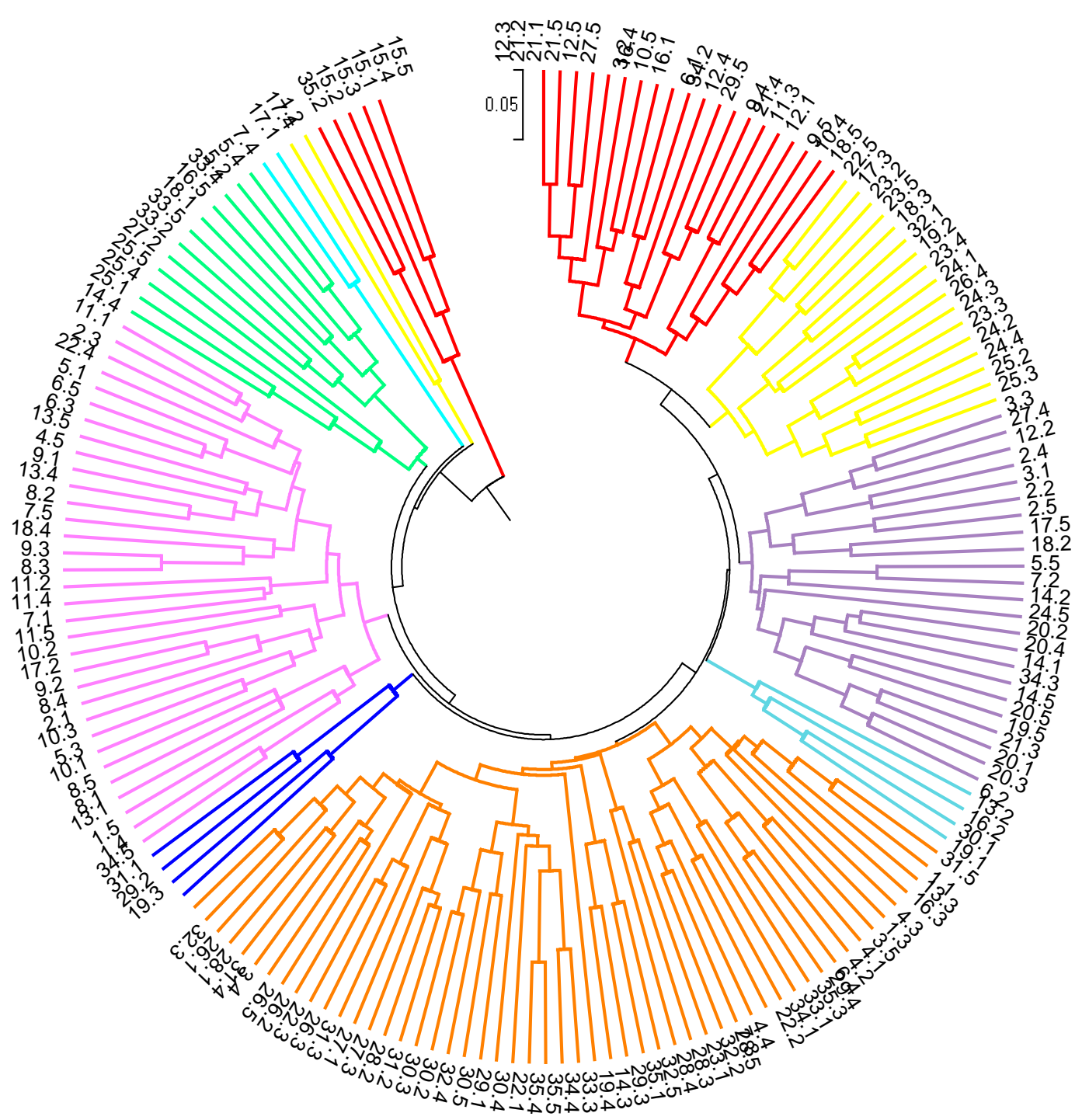

Figure 1- Cluster analysis of 175 accessions of popcorn based on UPGMA difference index through SSR markers polymorphism

\section{Conclusions}

Local popcorn populations are known to be richer in their genetic diversity than their hybrid counterparts. The use of genotypes in different groups in the breeding studies and having high evolution is beneficial in obtaining healthier and more effective results. At each stage of the study, examining genetic differences or richness within and between populations were observed high differences in each parameter. It was concluded that these differences would be an important resource of providing with a wide crop variety in terms of breeding and researchers.

This study was be first time for reveal of genome map of the genetic diversity on popcorn populations in Turkey. In addition, this study will give direction local hybrid seed production.

\section{Acknowledgements}

This study was supported by the Scientific Research Projects Unit of the Kahramanmaras Sutcu Imam University (Project number: 2014/3-27 D). 


\section{References}

Aci M M, Revilla P, Morsli A, Djemel A, Belalia N, Kadri Y, Khelifi-Saloui M, Ordás B \& Khelifi L (2013). Genetic diversity in Algerian maize (Zea mays L.) landraces using SSR markers. Maydica 58: 304-310

Adjanohoun A, Allagbe M, Noumavo P A, Gotoechan-Hodonou H, Sikirou R, Dossa K K, GleleKakaï R, Kotchoni S O \& Baba-Moussa L (2011). Effects of plant growth promoting rhizobacteria on field grown maize. Journal of Animal \& Plant Sciences 11(3): 1457-1465

Atanda A S \& Olaoye G (2017). Multiplex-Ready PCR assay of SSR marker diversity among quality protein maize inbred parental lines. South African Journal of Plant and Soil 34(2): 149-154 https://doi.org/10.1080/02571862.2016.1211325

Comertpay G (2008). Characterization of open polinated Turkish maize populations using morphological traits and SSRs molecular markers. Cukurova University of Cukurova Institute of Natural and Applied Science Field Crops Department, PhD Thesis, Adana (Published)

Doyle J J \& Doyle J L (1987). A rapid DNA isolation procedure for small quantities of fresh leaf tissue. Phytochemical Bulletin v.19 pp.11-15

Eschholz T W, Stamp P, Peter R, Leipner J \& Hund A (2010). Genetic structure and history of swiss maize (Zea mays L. ssp. mays) landraces. Genetic Resources and Crop Evolution 57(1): 71-84 https://doi.org/10.1007/s10722-009-9452-0

Frankel O H (1972). The significance, utilization and conservation of crop genetic resources. FAO, Rome

Gauthier P, Gouesnard B, Dallard J, Redaelli R, Rebourg C, Charcosset A, Boyat A (2002). RFLP diversity and relationships among traditional european maize populations. Theoretical and Applied Genetics 105(1): 91-99 https://doi.org/10.1007/s00122-002-0903-7

Kimura M \& Crow J F (1964). The Number of alleles that can be maintained in a finite population. Genetics 49(4): 725

Laborda P R, Oliveira K M, Garcia A A F, Paterniani M E A G Z, De Souza A P (2005). Tropical maize germplasm: What can we say about its genetic diversity in the light of molecular markers? Theoretical and Applied Genetics 111(7): 1288-1299 https://doi.org/10.1007/s00122-005-0055-7

Lewontin R C (1972). The Apportionment of human diversity. Evolutionary Biology 6: 381-398

Liu W S, Dong M, Song Z P, Wei W (2009). Genetic diversity pattern of Stipapurpurea populations in the hinterland of Qinghai-Tibet Plateau. Annals of Applied Biology 154: 57-65 https://doi.org/10.1111/j.1744-7348.2008.00274.x

McDermott J M \& McDonald B A (1993). Gene flow in plant pathosystems. Annual Review of Phytopathology 31(1): 353-373

Molin D, Coelho C J, Máximo D S, Ferreira F S, Gardingo J R, Matiello R R (2013). Genetic diversity in the germplasm of tropical maize landraces determined using molecular markers. Genetics and Molecular Research 12(1): 99-114

Nei M (1972). Genetic distance between populations. The American Naturalist 106(949): 283

Nei M (1973). Analysis of gene diversity in subdivided populations. Proceedings of the National Academy of Sciences 70(12): 3321

Nei M (1978). The theory of genetic distance and evolution of human races. Japanese Journal of Human Genetics 23(4): 341

Ribeiro C A G, Pinto M D O, Maciel T E F, Pastina M M, Barros E G D, Guimarães C T (2017). Universal tail sequence-SSR applied to molecular characterization of tropical maize hybrids. Scientia Agricola 74(2): 163-168 https://doi.org/10.1590/1678-992x-2016-0079

Rohlf F J (1992). Program numerical taxonomy and multivariate analysis system. Version 1.70 New York

Sharma L, Prasanna B M, Ramesh B (2010). Analysis of phenotypic and microsatellite-based diversity of maize landraces in India, especially from the North East Himalayan region. Genetica 138(6): 619-631 https://doi.org/10.1007/s10709-010-9436-1

Tahir N A \& Maeruf M S (2016). Assessment of salinity tolerance and SSR profile differentiation in nine maize genotypes (Zea mays L). Maydica 61 M18: 1-8

Vivodík M, Gálová Z, Balážová Ž, Petrovičová L (2017). Genetic variation of European maize genotypes (Zea mays L.) detected using SSR markers. Potravinarstvo Slovak Journal of Food Sciences 11(1): 126-131

Warburton M L, Ribaut J M, Franco J, Crossa J, Dubreuil P, Betrán FJ (2005). Genetic characterization of 218 elite CIMMYT inbred maize lines using RFLP markers. Euphytica 142:97-106 https://doi.org/10.1007/s10681-005-0817-y

Yao Q L, Yang K C, Pan G T, Rong T Z (2008). Genetic diversity of maize (Zea mays L.) landraces from southwest China based on SSR data. J. Genet. Genomics 34: 851-860 https://doi.org/10.1016/S1673-8527(07)60096-4

Zhang J, Guo J, Liu Y, Zhang D, Zhao Y, Zhu L, Huang Y, Zhang Z, Chen J (2016). Genome-wide association study identifies genetic factors for grain filling rate and grain drying rate in maize. Euphytica 212(2): 201-21 https://doi.org/10.1007/s10681-016-1756-5

(C) 2021 by the authors. Licensee Ankara University, Faculty of Agriculture, Ankara, Turkey. This article is an open access article distributed under the terms and conditions of the Creative Commons Attribution (CC BY) license (http://creativecommons.org/licenses/by/4.0/). 